\title{
Characterization of Graves' disease development after partial thyroidectomy for thyroid cancer
}

\author{
Huan Wan', Yingqiang Zhang' ${ }^{2}$, Yonghui Chen ${ }^{2}$ \\ ${ }^{1}$ PET-CT Center, National Cancer Center/Cancer Hospital, Chinese Academy of Medical Sciences and Peking Union Medical College, Beijing, \\ China; ${ }^{2}$ Department of Nuclear Medicine, Peking Union Medical College Hospital, Chinese Academy of Medical Sciences and Peking Union \\ Medical College, Beijing, China \\ Contributions: (I) Conception and design: H Wan, Y Chen; (II) Administrative support: Y Chen; (III) Provision of study materials or patients: Y \\ Zhang; (IV) Collection and assembly of data: H Wan, Y Zhang; (V) Data analysis and interpretation: H Wan, Y Zhang; (VI) Manuscript writing: All \\ authors; (VII) Final approval of manuscript: All authors. \\ Correspondence to: Yonghui Chen. Department of Nuclear Medicine, Peking Union Medical College Hospital, No. 1 Shuaifuyuan, Dongcheng \\ District, Beijing 100730, China. Email: chenyufirst@sina.com.
}

Background: Graves' disease (GD) is the most common cause of hyperthyroidism, and it rarely develops in patients who undergo partial thyroid removal for thyroid cancer. The present study characterized and analyzed the GD development mechanism after partial thyroidectomy for thyroid cancer and suggested optimized treatments for the condition.

Methods: A retrospective study from January 2013 to March 2017 was conducted in patients where GD occurred after partial thyroidectomy for thyroid cancer. Reviewed laboratory data included free 3,5,3'-triiodothyronine, free thyroxine, thyroid-stimulating hormone (TSH), thyroglobulin ( $\mathrm{Tg}$ ), anti- $\mathrm{Tg}$ antibody, anti-peroxidase antibody, and TSH receptor antibody levels. All laboratory indicators included preoperative and postoperative data. In addition, we collected patient neck ultrasound examination records and radioiodine (RAI) uptake test data.

Results: Among 5,600 patients diagnosed with GD, $5(0.09 \%)$ met the inclusion criteria and were enrolled. Patient mean age was $47.4 \pm 9.6$ years, and all were female with a papillary thyroid carcinoma stage I diagnosis. The interval between partial thyroidectomy and hyperthyroidism onset ranged from 2 to 25 months. We proposed possible mechanisms for this medical condition and identified several factors accelerating the disease course. To treat GD, a low RAI $(30 \mathrm{mCi})$ dose was prescribed to patients. All exhibited hypothyroidism at a 3-or 6-month follow-up, with stable clinical status at 1-year follow-up.

Conclusions: GD rarely develops after partial thyroidectomy for thyroid cancer. We recommend a low RAI dose to manage GD and ablate the remnants of thyroid cancer.

Keywords: Thyroidectomy; hyperthyroidism; thyroid cancer; radioiodine treatment; Graves' disease (GD)

Submitted Mar 24, 2021. Accepted for publication Jun 21, 2021.

doi: $10.21037 /$ tcr-21-501

View this article at: https://dx.doi.org/10.21037/tcr-21-501

\section{Introduction}

Graves' disease (GD) is a thyroid disease associated with multiple morbidity factors. The main cause is the binding of autoantibodies to over activated thyroid-stimulating hormone (TSH) receptors on thyroid follicular cells (1). In iodine-sufficient areas, GD is the most common cause of hyperthyroidism (2). Further research on the causes of GD will provide more guidance for clinical treatment.

The role of thyroid cancer in GD pathogenesis is unclear. Currently, the relationship between both is controversial, with GD pathogenesis after thyroid cancer treatment requiring further investigation. Partial thyroidectomy is a treatment for certain thyroid cancers. GD has been reported 
after partial thyroidectomy, although the incidence is low. Thus far, only 13 cases have been reported by previous studies, and none were reported in China (3-8). Once the condition occurs, GD seriously affects the quality of life of patients.

In the present study, we reported five rare GD cases that developed after thyroid lobectomy or partial thyroidectomy for thyroid cancer in a Chinese population. In addition, we reviewed and characterized all cases in the literature and proposed possible mechanisms and risk factors for this rare medical condition. Thus, our study provides valuable clinical data and insights for this condition. We present the following article in accordance with the STROBE reporting checklist (available at https://dx.doi.org/10.21037/tcr-21-501).

\section{Methods}

\section{Patients}

The study was conducted in accordance with the Declaration of Helsinki (revised in 2013). The study protocol was approved by the Institutional Review Board of the Ethics Committee of Peking Union Medical College, Beijing, China (No. S-K396). We retrospectively screened the patient database of the Department of Nuclear Medicine at Peking Union Medical College Hospital from January 2013 to March 2017. We reviewed data from patients with hyperthyroidism. Informed consent was taken from all patients.

\section{Inclusion criteria}

(I) Patients with thyroid cancer who underwent prior partial thyroidectomy or thyroid lobectomy instead of total thyroidectomy;

(II) Pathological classification performed according to the World Health Organization guidelines;

(III) Blood tests and technetium scintigraphy confirmed GD onset;

(IV) Patients with a complete medical history and clinical pathological data at diagnosis and follow-up.

\section{Exclusion criteria}

(I) Patients with a history of hyperthyroidism or abnormal thyroid function prior to thyroid surgery;

(II) Patients with a history of secondary malignant tumors or cervical surgeries prior to thyroid surgery;
(III) Metastasis or recurrence of thyroid carcinoma during follow-up;

(IV) Patients with serious diseases affecting the endocrine system or life span.

\section{Methodology}

We reviewed patient's age, sex, treatment regimen after partial thyroidectomy or lobectomy, the time interval between surgery and GD onset, and the time interval between surgery and radioiodine (RAI) treatment. Thyroid cancer staging was determined based on the American Joint Committee on Cancer/Tumor-Node-Metastasis Staging System for Differentiated and Anaplastic Thyroid Cancer, 8th edition (9). Reviewed laboratory data included free 3,5,3'-triiodothyronine, free thyroxine, TSH, thyroglobulin (Tg), anti-Tg antibody, anti-peroxidase antibody, and TSH receptor antibody (TRAb) levels. All laboratory indicators included preoperative and postoperative data. In addition, we collected patient neck ultrasound examination data and RAI uptake test data.

\section{Results}

In our database, 5,600 patients were diagnosed with hyperthyroidism during the study period. Only $5(0.09 \%)$ patients met the inclusion criteria. All were female with an average age of $47.4 \pm 9.6$ years and diagnosed with stage I papillary thyroid carcinoma (PTC) (Table 1).

We noted that the mean interval between partial thyroidectomy and hyperthyroidism onset was $12.0 \pm 9.7[2-25]$ months. Four patients showed typical clinical hyperthyroidism manifestations (e.g., palpitations, fatigue, tremors, anxiety, restless sleep, weight loss, heat intolerance, and sweating), whereas one patient (case 4) was asymptomatic throughout the treatment course. All patients underwent a RAI uptake test and showed normal or elevated RAI uptake in the cervical region.

We administered low-dose RAI treatments $(30 \mathrm{mCi})$ to all patients (Table 1) and examined TRAb titers after this treatment. Only one case (case 4) showed positive TRAb findings before surgery. These 5 patients received a 1-year follow-up, no one was lost to follow-up, and no recurrence of GD was observed.

\section{Case 1}

A 37-year-old woman was treated for palpitation and 
Table 1 General and clinical characteristics of enrolled patients

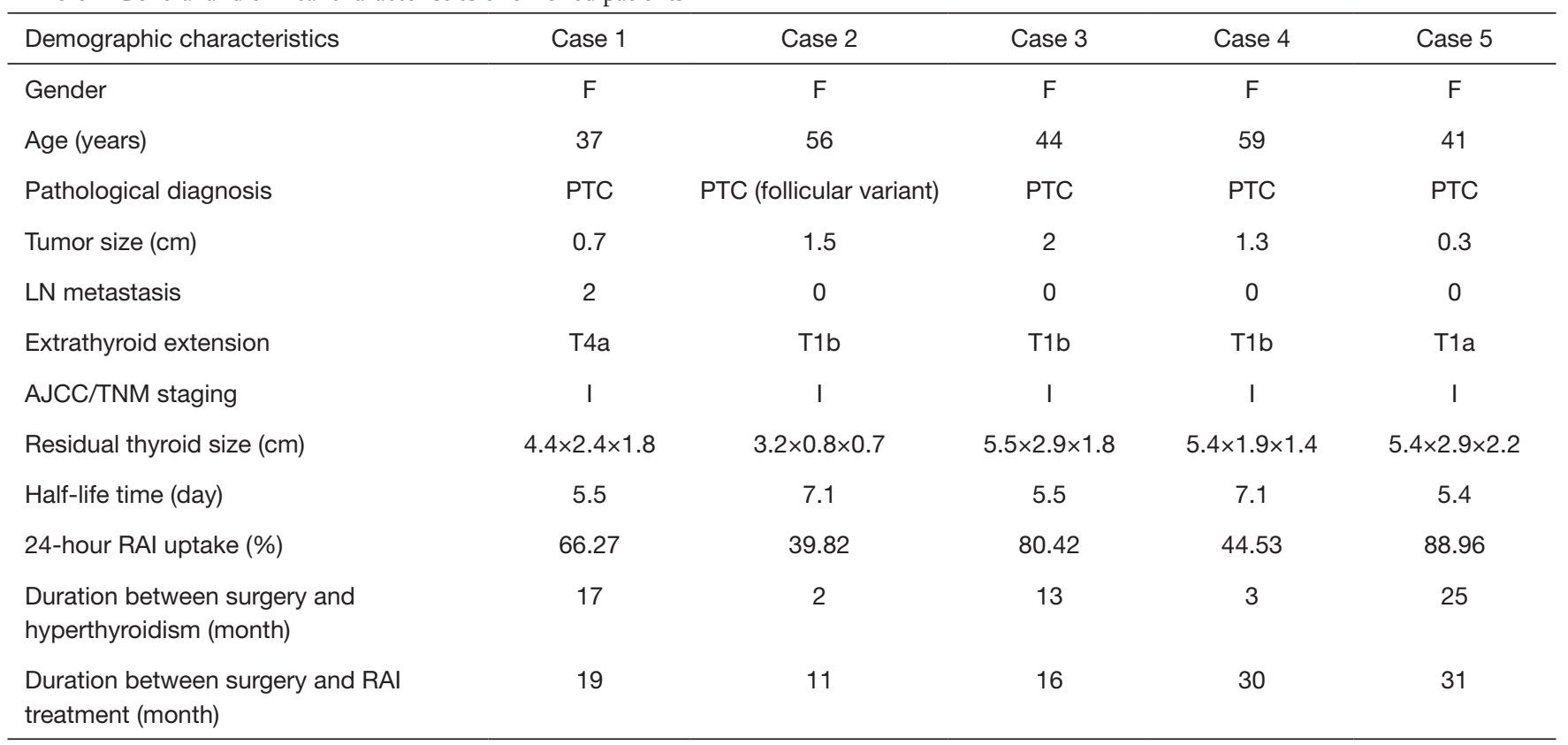

F, female; PTC, papillary thyroid carcinoma; LN, lymph node; AJCC/TNM, American Joint Committee on Cancer/Tumor-Node-Metastasis; $\mathrm{RAI}$, radioiodine.

Table 2 Case 1 laboratory data before and after RAI therapy

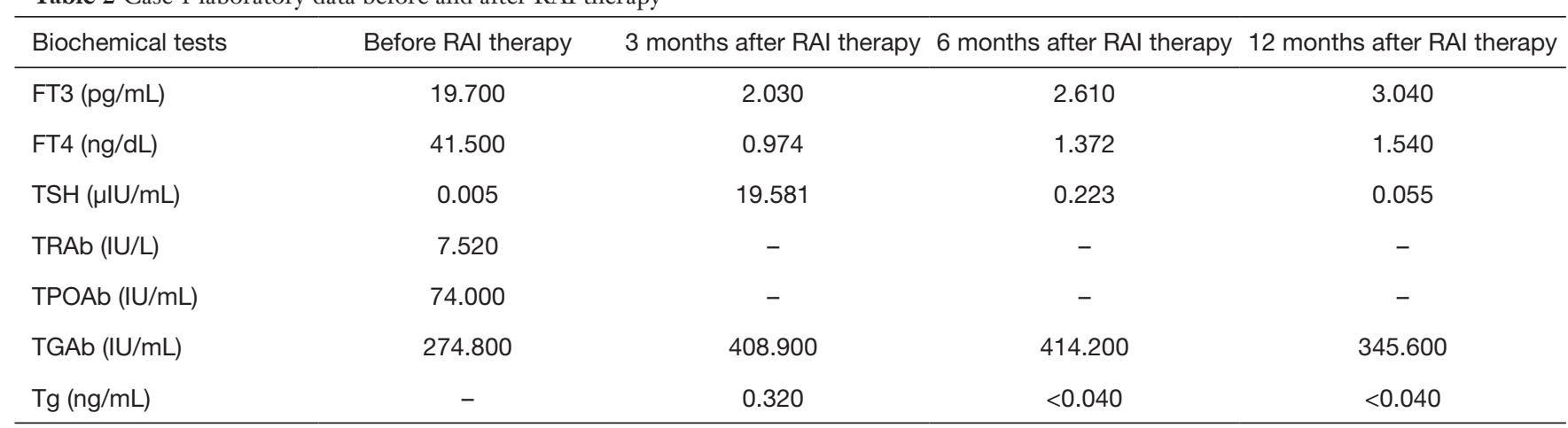

Normal range: FT3 1.8-4.1 pg/mL, FT4 0.81-1.89 ng/dL, TSH 0.38-4.34 $\mu \mathrm{lU} / \mathrm{mL}$, TRAb 0-1.75 IU/L, TPOAb <34 IU/mL, TGAb <115 IU/mL, Tg 1.4-78 ng/mL. RAl, radioiodine; FT3, free 3,5,3'-triiodothyronine; FT4, free thyroxine; TSH, thyroid-stimulating hormone; Tg, thyroglobulin; TGAb, anti-thyroglobulin antibody; TPOAb, anti-peroxidase antibody; TRAb, TSH receptor antibody.

fatigue symptoms. Seventeen months prior to her initial appointment, she underwent a right lobectomy of the thyroid with a dissection of right neck lymph nodes in levels II, III, IV, and VI for stage I PTC. She was diagnosed with GD based on laboratory findings and neck ultrasonography. She was considered clinically euthyroid before surgery. RAI therapy $(30 \mathrm{mCi})$ was used to treat her GD symptoms. Three months after RAI treatment, thyroid function tests showed that she had hypothyroidism. The patient then received levothyroxine replacement therapy. She did not demonstrate any sign of recurrence at 1-year follow-up (Table 2).

\section{Case 2}

A 56-year-old woman presented with initial symptoms of palpitations and weight loss. Two months prior to her appointment, she underwent a left lobectomy of the 
Table 3 Case 2 laboratory tests before and after RAI therapy

\begin{tabular}{|c|c|c|c|c|}
\hline Biochemical tests & Before RAI therapy & 3 months after RAI therapy & 6 months after RAI therapy & 12 months after RAI therapy \\
\hline FT4 (ng/dL) & 1.336 & 0.357 & 1.815 & 0.360 \\
\hline TSH $(\mu \mathrm{IU} / \mathrm{mL})$ & 0.004 & 93.680 & 0.009 & 98.608 \\
\hline TRAb (IU/L) & 4.580 & 10.010 & 7.980 & 0.450 \\
\hline TGAb (IU/mL) & 37.610 & 69.020 & 24.290 & 18.410 \\
\hline $\operatorname{Tg}(\mathrm{ng} / \mathrm{mL})$ & 19.720 & - & 1.550 & 0.690 \\
\hline
\end{tabular}

Normal range: FT3 1.8-4.1 pg/mL, FT4 0.81-1.89 ng/dL, TSH 0.38-4.34 $\mu \mathrm{IU} / \mathrm{mL}$, TRAb 0-1.75 IU/L, TPOAb $<34$ IU/mL, TGAb <115 IU/mL, Tg 1.4-78 ng/mL. RAl, radioiodine; FT3, free 3,5,3'-triiodothyronine; FT4, free thyroxine; TSH, thyroid-stimulating hormone; Tg, thyroglobulin; TGAb, anti-thyroglobulin antibody; TPOAb, anti-peroxidase antibody; TRAb, TSH receptor antibody.

Table 4 Case 3 laboratory tests before and after RAI therapy

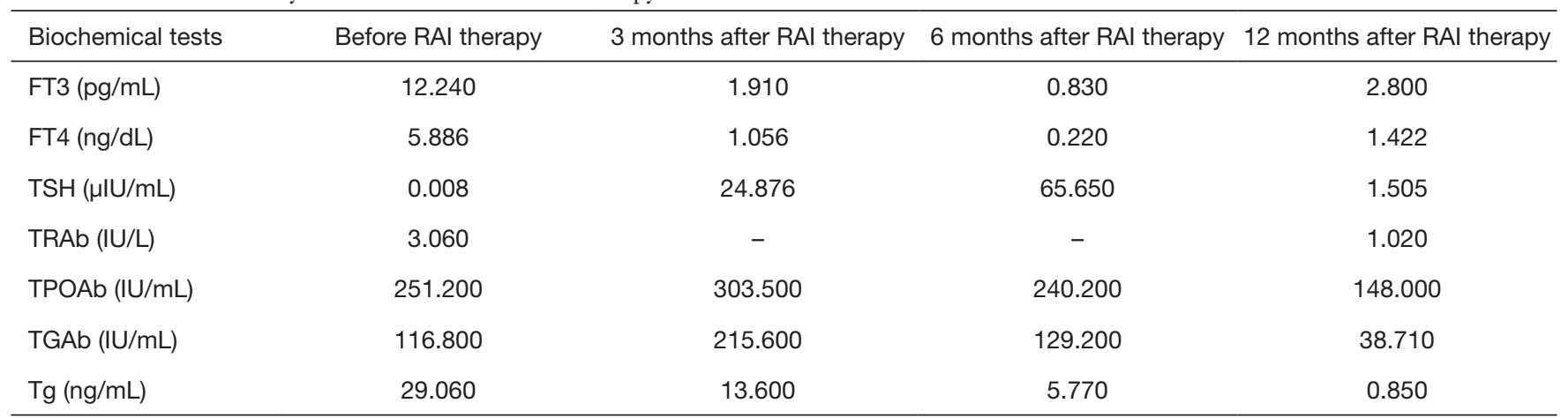

Normal range: FT3 1.8-4.1 pg/mL, FT4 0.81-1.89 ng/dL, TSH 0.38-4.34 $\mu \mathrm{lU} / \mathrm{mL}$, TRAb 0-1.75 IU/L, TPOAb <34 IU/mL, TGAb <115 IU/mL, Tg 1.4-78 ng/mL. RAl, radioiodine; FT3, free 3,5,3'-triiodothyronine; FT4, free thyroxine; TSH, thyroid-stimulating hormone; Tg, thyroglobulin; TGAb, anti-thyroglobulin antibody; TPOAb, anti-peroxidase antibody; TRAb, TSH receptor antibody.

thyroid with a dissection of left neck lymph nodes for a stage I follicular variant of PTC. She was considered clinically euthyroid before surgery. She was treated with RAI $(30 \mathrm{mCi})$. Three months after RAI treatment, she developed hypothyroidism and then received levothyroxine replacement. During the 1-year follow-up, she was stable without evidence of recurrence (Table 3).

\section{Case 3}

A 44-year-old woman had mainly palpitations and heat intolerance symptoms. Thirteen months prior to her visit, she received a partial thyroidectomy with a dissection of left neck lymph nodes in level VI for stage I PTC. She was considered clinically euthyroid before surgery. The patient started RAI treatment $(30 \mathrm{mCi})$ at the appointment.
Three months after receiving RAI treatment, she developed hypothyroidism and began receiving levothyroxine replacement. At the 1-year follow-up, she was doing well without evidence of recurrence (Table 4).

\section{Case 4}

A 59-year-old woman showed abnormal thyroid function 3 months after undergoing thyroidectomy for stage I PTC. She was considered clinically euthyroid before surgery, but laboratory tests revealed positive TRAb levels. She was diagnosed with GD 28 months after surgery. She then began to receive RAI treatment $(30 \mathrm{mCi})$. Three months after RAI treatment, she developed hypothyroidism and began to receive levothyroxine replacement. She had a stable clinical course at the 1-year follow-up (Table 5). 
Table 5 Case 4 laboratory tests before and after RAI therapy

\begin{tabular}{|c|c|c|c|c|}
\hline Biochemical tests & Before RAI therapy & 3 months after RAI therapy & 6 months after RAI therapy & 12 months after RAI therapy \\
\hline FT4 (ng/dL) & 1.478 & 0.341 & 1.338 & 0.180 \\
\hline TSH $(\mu \mathrm{IU} / \mathrm{mL})$ & 0.008 & 83.558 & 0.040 & 87.938 \\
\hline TRAb (IU/L) & 4.880 & 2.060 & 0.630 & 2.660 \\
\hline TGAb (IU/mL) & 41.000 & 928.600 & $1,226.000$ & $1,343.000$ \\
\hline $\operatorname{Tg}(\mathrm{ng} / \mathrm{mL})$ & 0.160 & 0.220 & $<0.040$ & $<0.040$ \\
\hline
\end{tabular}

Normal range: FT3 1.8-4.1 pg/mL, FT4 0.81-1.89 ng/dL, TSH 0.38-4.34 $\mu \mathrm{IU} / \mathrm{mL}$, TRAb 0-1.75 IU/L, TPOAb <34 IU/mL, TGAb <115 IU/mL, Tg 1.4-78 ng/mL. RAl, radioiodine; FT3, free 3,5,3'-triiodothyronine; FT4, free thyroxine; TSH, thyroid-stimulating hormone; Tg, thyroglobulin; TGAb, anti-thyroglobulin antibody; TPOAb, anti-peroxidase antibody; TRAb, TSH receptor antibody.

Table 6 Case 5 laboratory tests before and after RAI therapy

\begin{tabular}{lcc}
\hline Biochemical tests & $\begin{array}{c}3 \text { months after RAI } \\
\text { therapy }\end{array}$ & $\begin{array}{c}6 \text { months after RAI } \\
\text { therapy }\end{array}$ \\
\hline FT3 $(\mathrm{pg} / \mathrm{mL})$ & 1.830 & 0.470 \\
$\mathrm{FT4}(\mathrm{ng} / \mathrm{dL})$ & 1.300 & 0.253 \\
$\mathrm{TSH}(\mu \mathrm{lU} / \mathrm{mL})$ & $<0.008$ & 62.095 \\
TRAb $(\mathrm{IU} / \mathrm{L})$ & - & 2.57 \\
$\mathrm{TPOAb}(\mathrm{IU} / \mathrm{mL})$ & $>600.000$ & 535.100 \\
$\mathrm{TGAb}(\mathrm{IU} / \mathrm{mL})$ & 339.100 & 283.300 \\
$\mathrm{Tg}(\mathrm{ng} / \mathrm{mL})$ & 0.140 & 0.200 \\
\hline
\end{tabular}

Normal range: FT3 $1.8-4.1 \mathrm{pg} / \mathrm{mL}$, FT4 $0.81-1.89 \mathrm{ng} / \mathrm{dL}$, TSH 0.38-4.34 $\mathrm{IJU} / \mathrm{mL}$, TRAb $0-1.75 \mathrm{IU} / \mathrm{L}$, TPOAb $<34 \mathrm{IU} / \mathrm{mL}$, TGAb $<115 \mathrm{IU} / \mathrm{mL}$, Tg $1.4-78 \mathrm{ng} / \mathrm{mL}$. RAl, radioiodine; FT3, free 3,5,3'-triiodothyronine; FT4, free thyroxine; TSH, thyroidstimulating hormone; Tg, thyroglobulin; TGAb, anti-thyroglobulin antibody; TPOAb, anti-peroxidase antibody; TRAb, TSH receptor antibody.

\section{Case 5}

This case involved a 41-year-old woman whose main complaints were palpitations and insomnia. She had a right lobectomy of the thyroid for stage I PTC, 31 months prior to her presentation. Laboratory workup demonstrated hyperthyroidism, and she was diagnosed with GD. She was subsequently treated with RAI $(30 \mathrm{mCi})$. Three months after receiving RAI treatment, she showed subclinical hyperthyroidism. At the 6-month follow-up, she demonstrated evidence of hypothyroidism and was treated with levothyroxine. During the 1-year follow-up, her clinical symptoms were well controlled (Table 6).

\section{Discussion}

GD is an autoimmune thyroid disorder with a multifactorial etiology (2). Patients with GD typically present with thyroid enlargement, palpitations, tachycardia, tremulousness, heat intolerance, weight loss, and anxiety (1). Both genetic and non-genetic factors play significant roles in the etiopathology mechanisms of GD. Genetic risk factors are associated with immune-regulatory genes and thyroid autoantigens (e.g., Tg and TSH receptor genes) $(10,11)$. Non-genetic risk factors also contribute to GD initiation and progression and include infection, thyroid damage, psychological stress, smoking, and the female gender (11-13). Owing to the markedly higher prevalence of GD in women, sex hormones and skewed inactivation of the $\mathrm{X}$ chromosome are suspected as important triggers $(2,11)$. A GD diagnosis is established based on clinical symptoms, elevated thyroxine and triiodothyronine levels and low TSH levels (11). Additionally, TRAb titers, RAI uptake, and thyroid ultrasound with Doppler can be adjunctively used to confirm a GD diagnosis (11).

Patients with GD have an elevated risk of developing thyroid cancer (14). The incidence of this cancer in patients with GD is approximately $2-10 \%$, with $88 \%$ of cases being PTC (14-16). However, it is rare for patients with thyroid cancer to develop GD after partial thyroidectomy (3-8). In our study, the incidence of GD development from thyroid carcinoma was $0.09 \%$. Thus far, only 13 cases diagnosed as GD after thyroidectomy for thyroid nodules or thyroid cancer have been reported in previous studies, with none from China (3-8). The mechanism whereby GD develops after partial thyroidectomy for thyroid cancer is still unclear; thus, treatments for GD after partial thyroidectomy require 
further investigation.

We reviewed the five selected cases at our hospital and also analyzed eight previously reported cases (5-7). We divided these cases into three groups: (I) TRAb positive before surgery; (II) TRAb negative before surgery, and (III) TRAb unknown before surgery. When we studied these groups more closely, we observed that cases in the TRAb positive before surgery group (Group 1) had a short GD occurrence time that possibly suggested that being TRAb positive before surgery may have accelerated GD occurrence. The TRAb negative before surgery group (Group 2) showed that although TRAb titers were negative before surgery, GD subsequently developed with TRAb titers becoming positive when GD was diagnosed. Thus, TRAb titers may reflect an important mechanism during GD development (Table 7).

Previous studies have suggested that pathogenesis may be related to changes in immune status caused by surgery. Thyroid surgery may contribute to GD initiation by altering immunological homeostasis. Several hypotheses have been proposed. In patients whose TRAb antibody titers were already elevated prior to thyroid surgery, abnormalities in antigen-presenting cells may have altered the immune system, leading to hyperactivity of the thyroid gland (7). In patients with normal or lower TRAb titers, thyroid surgery may have destroyed thyroid epithelial cells and released antigens. These antigens may have stimulated helper T cells, leading to autoantibody generation (7).

Other factors may influence GD initiation and progression. In all cases reported since 1997, only one patient was male. Females have stronger immune responses and are more vulnerable to autoimmune diseases (17); sex hormones and other steroid hormones exert immunoregulatory effects during the development of autoimmune diseases (17). In addition, histopathological thyroid cancer subtypes may contribute to GD susceptibility. Two cases (2 and 4) in our cohort had a very short interval between surgery and hyperthyroidism onset, and one case was diagnosed as a follicular variant of PTC. Different surgical procedures did not appear to affect GD development.

At present, relevant treatment options for GD after lobectomy or partial thyroidectomy are rarely reported. We must simultaneously consider the recurrence rate and metastatic risk for thyroid cancer while treating GD. To improve clinical treatment results, further research must be conducted. The current mainstays for GD treatment include anti-thyroid drugs, RAI therapy, and surgery. RAI therapy is safe and cost-effective and is used as the firstline treatment for GD, although there are some regional differences (2). A low RAI dose $(30 \mathrm{mCi})$ is adequate to ablate thyroid remnants after partial thyroid removal for well-differentiated thyroid cancer. This treatment regimen carries a low risk for radiation thyroiditis (18). In patients without contraindications, RAI therapy is the most optimal treatment for those who develop GD after surgical treatment for thyroid cancer. Anti-thyroid agents (e.g., propylthiouracil, thiamazole, and carbimazole) are typically used to restore euthyroidism before surgery or RAI therapy (2). When RAI therapy and surgery are contraindicated, anti-thyroid agents have significant potential side effects when used over the long term (2). Surgery is the most efficient way to treat GD, but may not be the best treatment option for patients who have a history of other thyroid operations. Repeated surgical operations are associated with a higher incidence of complications (19). In our case-cohort, four patients exhibited hypothyroidism 3 months after applying RAI therapy, with the fifth patient at the 6-month follow-up. This indicated that RAI therapy was effective for GD. All cases maintained hypothyroidism at the 1-year follow-up with minimal side effects.

\section{Conclusions}

We reported five GD cases that occurred after partial thyroidectomy for PTC and proposed several hypotheses for the cause and development of this rare medical phenomenon. We shared our experiences in treating GD within this context. Our study also had limitations. Owing to condition rarity, we require more cases to fully explore underlying mechanisms; therefore, we will continue collecting similar cases and maintaining records for longer follow-up periods. 


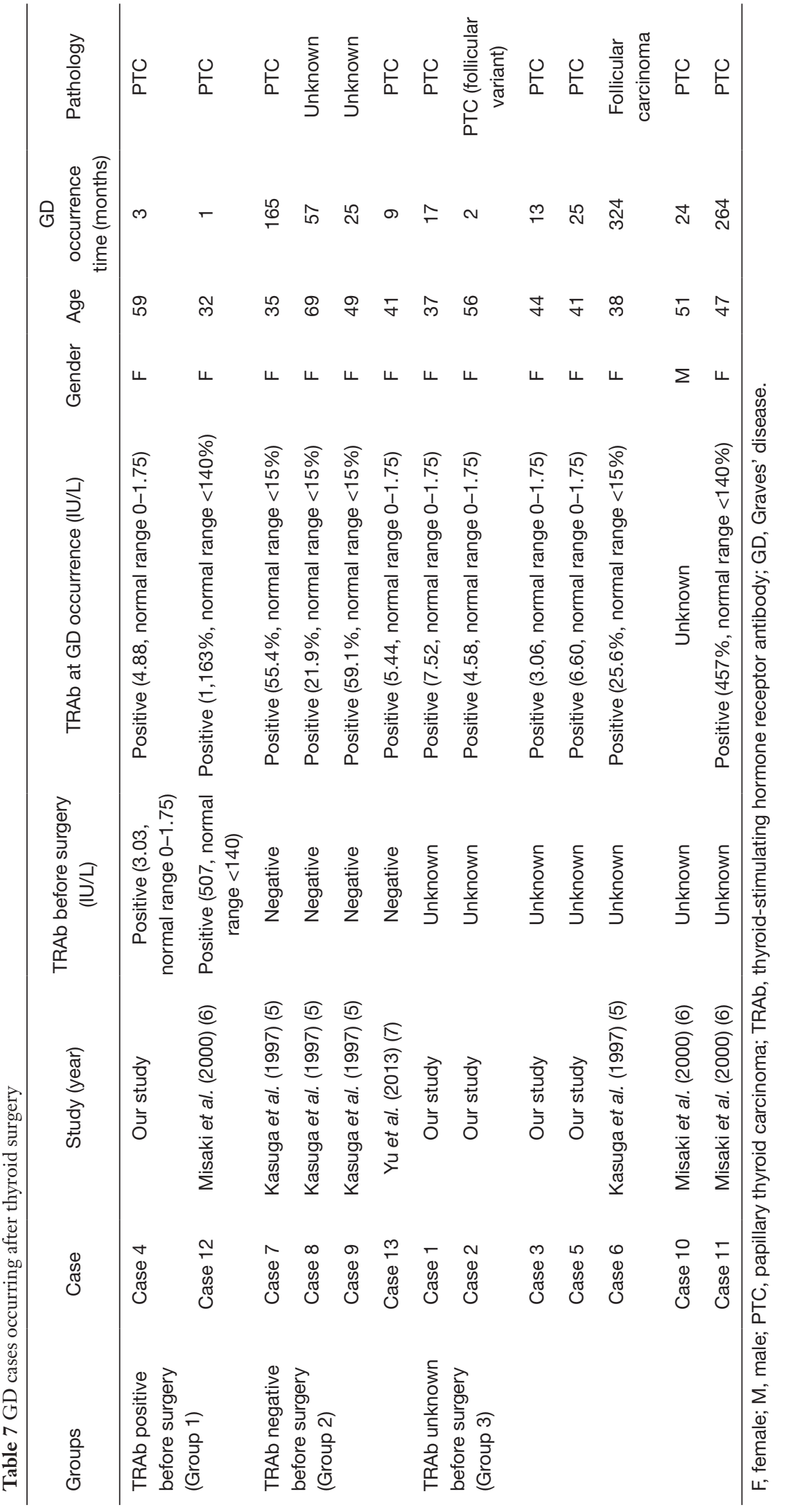




\section{Acknowledgments}

Funding: None.

\section{Footnote}

Reporting Checklist: The authors have completed the STROBE reporting checklist. Available at https://dx.doi. org/10.21037/tcr-21-501

Data Sharing Statement: Available at https://dx.doi. org/10.21037/tcr-21-501

Conflicts of Interest: All authors have completed the ICMJE uniform disclosure form (available at https://dx.doi. org/10.21037/tcr-21-501). The authors have no conflicts of interest to declare.

Ethical Statement: The authors are accountable for all aspects of the work in ensuring that questions related to the accuracy or integrity of any part of the work are appropriately investigated and resolved. The study was conducted in accordance with the Declaration of Helsinki (as revised in 2013). The current study protocol was approved by the Institutional Review Board of Ethics Committee of Peking Union Medical College, Beijing, China (No. S-K396). Written informed consent was obtained from all participants before enrolment.

Open Access Statement: This is an Open Access article distributed in accordance with the Creative Commons Attribution-NonCommercial-NoDerivs 4.0 International License (CC BY-NC-ND 4.0), which permits the noncommercial replication and distribution of the article with the strict proviso that no changes or edits are made and the original work is properly cited (including links to both the formal publication through the relevant DOI and the license). See: https://creativecommons.org/licenses/by-nc-nd/4.0/.

\section{References}

1. Burch HB, Cooper DS. Management of Graves Disease: A Review. JAMA 2015;314:2544-54.

2. De Leo S, Lee SY, Braverman LE. Hyperthyroidism. Lancet 2016;388:906-18.

3. Tamai H, Hirota Y, Matsuzuka F, et al. Three cases developed Graves' disease after surgical treatment for thyroid carcinomas. Horumon To Rinsho 1982;30:124-5.

4. Nagai K, Tamai H, Matsubayashi S, et al. A case developed Graves' disease after thyroid nodule. Horumon To Rinsho 1987;35:107-9.

5. Kasuga Y, Kobayashi S, Fujimori M, et al. Development of Graves' disease after surgical treatment for thyroid nodules: report of four cases. Endocr J 1997;44:567-70.

6. Misaki T, Iwata M, Kasagi K, et al. Hyperthyroid Graves disease after hemithyroidectomy for papillary carcinoma: report of three cases. Endocr J 2000;47:191-5.

7. Yu HM, Park SH, Lee JM, et al. Graves' Disease that Developed Shortly after Surgery for Thyroid Cancer. Endocrinol Metab (Seoul) 2013;28:226-30.

8. Umena S, Takano T, Iijima T, et al. A case of repeated painless thyroiditis followed by Graves' disease. Endocr J 1995;42:821-6.

9. Tuttle RM, Haugen B, Perrier ND. Updated American Joint Committee on Cancer/Tumor-Node-Metastasis Staging System for Differentiated and Anaplastic Thyroid Cancer (Eighth Edition): What Changed and Why? Thyroid 2017;27:751-6.

10. Dobyns BM, Sheline GE, Workman JB, et al. Malignant and benign neoplasms of the thyroid in patients treated for hyperthyroidism: a report of the cooperative thyrotoxicosis therapy follow-up study. J Clin Endocrinol Metab 1974;38:976-98.

11. Marinò M, Latrofa F, Menconi F, et al. Role of genetic and non-genetic factors in the etiology of Graves' disease. J Endocrinol Invest 2015;38:283-94.

12. Wiersinga WM. Smoking and thyroid. Clin Endocrinol (Oxf) 2013;79:145-51.

13. Winsa B, Adami HO, Bergström R, et al. Stressful life events and Graves' disease. Lancet 1991;338:1475-9.

14. Boutzios G, Vasileiadis I, Zapanti E, et al. Higher incidence of tall cell variant of papillary thyroid carcinoma in Graves' Disease. Thyroid 2014;24:347-54.

15. Ozaki O, Ito K, Kobayashi K, et al. Thyroid carcinoma in Graves' disease. World J Surg 1990;14:437-40; discussion 440-1.

16. Staniforth JUL, Erdirimanne S, Eslick GD. Thyroid carcinoma in Graves' disease: A meta-analysis. Int J Surg 2016;27:118-25.

17. Paavonen T. Hormonal regulation of immune responses. Ann Med 1994;26:255-8.

18. Lin JD, Chao TC, Huang MJ, et al. Use of radioactive iodine for thyroid remnant ablation in well-differentiated 
thyroid carcinoma to replace thyroid reoperation. Am J

Clin Oncol 1998;21:77-81.

19. Bal CS, Kumar A, Pant GS. Radioiodine lobar ablation as an alternative to completion thyroidectomy in patients with differentiated thyroid cancer. Nucl Med Commun 2003;24:203-8.
Cite this article as: Wan H, Zhang Y, Chen Y. Characterization

of Graves' disease development after partial thyroidectomy for thyroid cancer. Transl Cancer Res 2021;10(7):3168-3176. doi: $10.21037 /$ tcr-21-501 